\title{
Neurons and glia in close contact
}

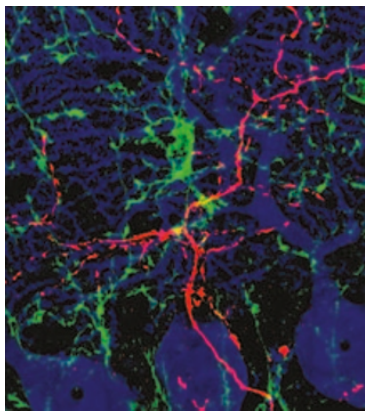

Purkinje cells labelled with anti-calbindin (blue), NG2 ${ }^{+}$cells labelled with anti-NG2 antibodies (green), and a climbing fibre labelled with Alexa-488 dextran (red) in the rat cerebellar cortex. Image courtesy of S. C. Lin, H. Nishiyama and D. E. Bergles, Department of Neuroscience, Johns Hopkins University School of Medicine, Baltimore, USA
Climbing fibres (CFs) from the inferior olive, one of the two main inputs to the cerebellum, make direct synaptic contacts with the cerebellum's output neurons, Purkinje cells. A new study shows that CFs also form conventional glutamatergic synapses with a population of glia in the cerebellar cortex. These glial cells, which express the proteoglycan NG2, might have an important part to play in the control of cerebellar function by $\mathrm{CF}$ inputs.

$\mathrm{NG} 2$-expressing $\left(\mathrm{NG}^{+}\right)$glia are oligodendrocyte precursor cells that are found throughout the developing and mature CNS. In the cerebellar cortex, they extend many processes around Purkinje cell dendrites. Like Bergmann glia, which ensheath excitatory synapses on Purkinje cells, NG2 ${ }^{+}$ cells express ionotropic glutamate receptors. But whereas Bergmann glia express uptake sites for glutamate and can, therefore, limit the spread of this neurotransmitter, $\mathrm{NG} 2^{+}$cells do not express glutamate transporters. What is the role of $\mathrm{NG}^{+}$cells in the cerebellum?

Lin and colleagues made whole-cell recordings from $\mathrm{NG}_{2}{ }^{+}$ cells in mouse cerebellar slices. These non-excitable cells responded to CF stimulation with a transient inward current mediated by calcium-permeable AMPA $(\alpha$-amino-3-hydroxy-5-methyl4-isoxazole propionic acid) receptors. The response seemed to be due to glutamate release at a conventional synapse rather than ectopic release, having rapid rise and decay kinetics and being remarkably stable with repeated stimulation. Paired recordings of Purkinje cells and $\mathrm{NG}^{+}$cells showed that one $\mathrm{CF}$ can provide input to both cell types; however, unlike Purkinje cells, $\mathrm{NG2}^{+}$cells were found to receive input from more than one CF.

In an electron microscopic analysis, conventional synaptic junctions were seen between CFs and the processes of physiologically characterized $\mathrm{NG}^{+}$cells. Synapses on $\mathrm{NG}_{2}{ }^{+}$cells were not wrapped by Bergmann glia, but the processes of $\mathrm{NG}^{+}$cells were found in direct apposition with Bergmann glial lamellae.

\section{PAIN}

\section{Great expectations}

Placebos have been shown to relieve pain - a phenomenon known as placebo analgesia. A new study by Petrovic and colleagues has found that placebos can also affect emotion, alleviating the impact of unpleasant experiences.

Placebo analgesia and reward processing have similar characteristics: both involve anticipation of a positive outcome and are highly dependent on expectation. The authors conjectured that as emotion is closely associated with reward, it might also be affected by placebos. To test this, they used the so-called 'expectation manipulation' paradigm, in which a treatment expectation was induced in participants one day before the test day, using drugs that have well-established specific effects on emotion (that is, anxiolytic drugs and their blockers).

On day 1, participants were asked to rate the unpleasantness of neutral and disturbing pictures that were shown to them with or without the administration of an anxiolytic drug and its blocker.
They were told in advance about the possible effect of the drug and its blockers on their perception of unpleasantness. As expected, the anxiolytic drug reduced the participants' perceived unpleasantness of the disturbing pictures, an effect that was reversed by administration of the drug's blocker. Neither the drug nor its blocker affected ratings of the neutral pictures.

On day 2, participants were told that the same treatments would be applied while their brains were scanned with functional MRI (fMRI). However, they were, in fact, treated with saline instead. The unpleasantness ratings of the disturbing pictures under the placebo condition (in which participants thought they had received the anxiolytic drug) were reduced by $29 \%$ compared with ratings under the control condition (in which participants thought they had received the drug followed by its blocker).

Interestingly, like placebo analgesia, the rostral anterior cingulate cortex (rACC) and the lateral orbitofrontal cortex (lObfc) - regions of the brain that are important in reward expectation - were activated by the emotional placebo. The increase in the activity of the rACC, but not that of the lObfc, correlated with the decrease in unpleasantness ratings of the pictures after the placebo treatment. In other words, the participants who reported the largest placebo response also showed the most extensive rACC activation.

These findings indicate that placebo analgesia - or the placebo effect in general - might be a special form of reward processing. This could shed new light on the evolutionary advantages of this powerful and fascinating phenomenon.

Jane Qiu

\section{(2) References and links}

ORIGINAL RESEARCH PAPER Petrovic, P. et al. Placebo in emotional processing - induced expectations of anxiety relief activate a generalized modulatory network. Neuron 46,957-969 (2005)

FURTHER READING Colloca, L. \& Benedetti, F. Placebos and painkillers: is mind as real as matter? Nature Rev. Neurosci. 6 545-552 (2005) | Vogt, B. Pain and emotion interactions in subregions of the cingulate cortex. Nature Rev. Neurosci. 6,533-544 (2005)

INGVAR'S WEB SITE

http://www.cns.ki.se/en/research/martin_ingvar/martin_ ingvar/ 